\title{
Time reversal seismic source imaging using peak average power ratio (PAPR) parameter
}

\author{
Anna Franczyk ${ }^{1}$ - Andrzej Leśniak ${ }^{1}$ Damian Gwiżdż ${ }^{1}$
}

Received: 20 February 2017 / Accepted: 21 February 2017/Published online: 7 March 2017

(c) The Author(s) 2017. This article is published with open access at Springerlink.com

\begin{abstract}
The time reversal method has become a standard technique for the location of seismic sources. It has been used both for acoustic and elastic numerical modelling and for $2 \mathrm{D}$ and $3 \mathrm{D}$ propagation models. Although there are many studies concerning its application to point sources, little so far has been done to generalise the time reversal method to the study of sequences of seismic events. The need to describe such processes better motivates the analysis presented in this paper. The synthetic time reversal imaging experiments presented in this work were conducted for sources with the same origin time as well as for the sources with a slight delay in origin time. For efficient visualisation of the seismic wave propagation and interference, a new coefficient-peak average power ratiowas introduced. The paper also presents a comparison of visualisation based on the proposed coefficient against a commonly used visualisation based on a maximum value.
\end{abstract}

Keywords Time reversal - Seismic wave modelling . Source location

Anna Franczyk

franczyk@geol.agh.edu.pl

Andrzej Leśniak

lesniak@agh.edu.pl

Damian Gwiżdż

damian.gwizdz@gmail.com

1 AGH University of Science and Technology, Krakow, Poland

\section{Introduction}

Measurement of seismic emissions related to natural or human activity is an important tool in the recognition of internal structures and the dynamic behaviour of rock mass. The energy, source location and focal mechanism of such emissions are analysed. The seismic source determines how seismic energy radiates through the rock mass, meaning it has a crucial role in the analysis of kinematic and dynamic changes in a given area.

One of the key parameters used in an analysis of these changes is the location of emission sources.

In mining practice, source location is performed separately for each event, independently of other recorded events. Another method is the relative location method, for which the location of the source is determined relative to the location of adjacent sources. There are several variants of this method (see, e.g., Waldhauser and Ellsworth 2000; Rudzinski and Debski 2012; Douglas 1967). In oint event location, events are relocated according to the location of the so-called "master event" (Douglas 1967). Relative location methods usually improve the accuracy of source location; however, they are strongly influenced by data quality, similarly to non-relative methods of location (Rudzinski and Debski 2011; Debski and Klejment 2016). The crucial factors are determination errors of the aforementioned parameters, like $\mathrm{P}$ or $\mathrm{S}$ wave onsets.

The superimposing of two or more events in a seismic records, together with the signal-to-noise ratio of the data and the velocity model, are considered as the most important factors that hamper not only event location, but also analysis of its source mechanism and energy estimation. The coda component of the first event usually has larger amplitudes than the $\mathrm{P}$ wave of the second wave. Thus, the evaluation of the second events' parameters that 
are needed for location is difficult or even impossible. Superposition of seismic events can happen for high energy-induced seismic emissions recorded in underground mines. Smaller, secondary events are recorded after the high energy event, and frequently overlap the main waveform. In such cases, it is only possible to locate the first event and the total energy of the group of events.

A promising solution in such cases could be utilisation of the whole waveform recorded by all the sensors (even for superimposed events) for source location. The time reversal imaging (TRI) technique utilises the fact that the wave equation is reversible in time, meaning that it does not change form when the time direction is reversed, for example $t \rightarrow-t$. This gives rise to the assumption that the observed wave propagation is symmetrical in time when energy dissipation is ignored. Positive orientation of the time axis allows the observation of the emission of waves from sources located beneath the surface and propagation toward sensors located on the surface. For negative time direction, the time-reversed recorded signals are the sources of energy in sensors' positions and back propagate through the rock mass to their sources. The energy is then focused at the point where the source is located. Similarly, for superimposed events from a few sources, time-reversed propagation from sensors focuses at the individual sources. The quality of the focusing depends largely on the knowledge of the velocity structure of the geological media where the wave propagates.

The idea of TRI was proposed in the 1960s by Parvulescu and Clay (1965) for submarine communication. These ideas were then further expanded by Fink (Fink et al. 1989; Fink 1992, 1997), who made TRI better known. The development of theoretical aspects of the method and its application in non-destructive testing were continued by Anderson et al. (2009a, b) and Saenger et al. (2011). The TRI technique in seismology has been used in source location and identification of source mechanisms (Gajewski and Tessmer 2005; Larmat et al. 2006, 2010; Kawakatsu and Montagner 2008; Steiner and Saenger 2010; Artman et al. 2010; Debski 2015). In seismic exploration the TRI technique has been applied to wave field migration (Baysal et al. 1983; McMechan 1983; Tarantola 1988; Fichtner et al. 2006) and structure imaging in complex geological conditions like salt domes (Willis et al. 2006). Most of these projects exploited TRI for an assumed single point source location. TRI methods have also been successfully applied to the location of multiple simultaneous sources (Saenger et al. 2011). However, according to previous results, exploration of the potential of TRI to detect the details of finite rupture processes does not provide encouraging results (Blomgren et al. 2002; Kremers et al. 2011). The main problem is caused by the limits of the source size that could be reconstructed (Anderson et al. 2011).

In the present paper, we discuss the possibilities of using TRI in the location of sequences of seismic events using the new imaging coefficient: peak average power ratio. The former definition of this parameter is presented in "Quantitative assessment of focusing for multiple source point simulations with the same excitation time". We use synthetic seismic data generated in stratified two-dimensional geological media. The modelled sequences consist of several events with the same origin time and with relatively small delays between them. In this work, a comparison between the location of seismic sources and a visualisation based on the proposed parameter and maximum absolute value is also presented.

The paper is organised as follows: In the second section, the methodology used for location of sequences of seismic events together with a description of the PAPR imaging parameters is presented. Subsequently, we present synthetic tests of the proposed imaging technique. Numerical modelling was performed for different source distribution and different temporal source emission. The first example simulated random distribution of seismic events. The second example simulated a deterministic case: a rupture process with sources laid out perpendicular to the receiver array and with a slight time lag between subsequent source emissions.

\section{Outline of the methodology}

For our TRI of the sequence of seismic events, we employ a finite difference algorithm to model wave propagation in $2 \mathrm{D}$ acoustic media. The location of source coordinates was performed in three steps based on the PARP coefficient value. In the first step, the synthetic seismograms for the given geological model and the exact locations of the source points and receivers were computed. In the second step of the computational algorithm, the full synthetic seismograms were reversed in time in order to perform the role of source functions. These seismograms were used in the backward propagation of the seismic wave that was the third stage of the TRI rupture process imaging experiment. The backward propagation was performed iteratively and at each time step of the propagation algorithm the value of the PARP coefficient was updated and stored.

\section{Forward simulation}

The goal of the forward simulation in our case was to create a synthetic dataset for acoustic emissions in the given geological model. Synthetic seismograms were 
computed for constant density using a two-dimensional acoustic wave field equation described by

$\frac{\partial^{2} P}{\partial t^{2}}=\kappa\left[\frac{1}{\rho}\left(\frac{\partial^{2}}{\partial x^{2}}+\frac{\partial^{2}}{\partial z^{2}}\right) P+\nabla \cdot f\right]$,

where $P(x, z)$ is the pressure field, $f$ denotes body force, $\rho=\rho(x, z)$ denotes density of the medium and $\kappa=\kappa(x$, $y$ ) is the bulk modulus.

The acoustic wave field equation was transformed to a system of first-order hyperbolic linear equations (Virieux 1986).

The equation was solved numerically using a staggeredgrid, finite difference method that is stable for all values of Poisson's ratio and characterised by relatively small grid dispersion and grid anisotropy (Levander 1988). In this work, second order temporal and fourth order spatial operators under Cartesian coordinates were used.

For semi-infinite space modelling, free surface boundary conditions at $z=0$ were applied. The other boundaries at the grid periphery were coded to satisfy the wave absorbing conditions (Cerjan et al. 1985). The source wavelet in the forward modelling was estimated with the Ricker wavelet, the second derivative of a Gaussian. We restricted modelling to non-dissipative media for simplicity.

\section{Source location reverse modelling}

The backward propagation of the seismic wave presented in this work is based on the same acoustic numerical modelling in two-dimensional space as the forward modelling. In contrast to standard seismic wave modelling, which uses seismic explosions as the initial boundary condition, time-reversed recordings are used in the backward propagation as the sources of seismic waves. A modelling scheme the same as the modelling scheme used in the first stage of computation is used to propagate the wave field backward into the assumed model. Assuming that the receiver configuration is sufficiently dense and regular, the time-reversed wave field will focus in space and time in the computational node corresponding to the coordinates of the source point. The focusing point should be noticeable as an area of abnormal values by visualisation of the seismic field obtained during backpropagation of the inversed seismograms.

\section{The imaging parameters: peak average power ratio coefficient and the maximum value}

The time reversal imaging methods that have so far been carried out are mostly based on the maximum value of a given parameter. The following were tested as imaging parameters: maximum horizontal and vertical displacement components (Hu and McMechan 1988; Steiner and Saenger 2012; Saenger 2011), maximum particle velocity (Steiner et al. 2008), strain components (Blomgren et al. 2002), maximum amplitude of pressure value (Gajewski and Tessmer 2005), stress components' energy density (Gajewski and Tessmer 2005; Saenger 2011), maximum P- and $\mathrm{S}$ wave energy density, maximum energy density, and (a)

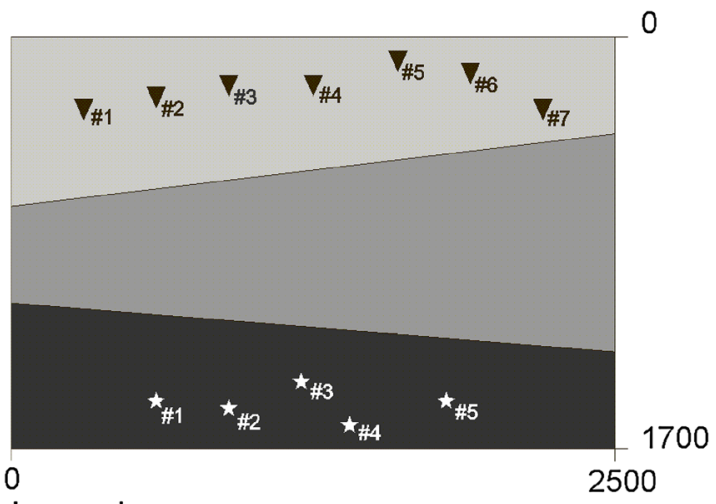

Legend:

$\nabla$ receiver

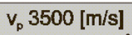
p $3500\left[\mathrm{~kg} / \mathrm{m}^{3}\right]$ is source

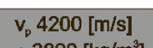
p $3800\left[\mathrm{~kg} / \mathrm{m}^{3}\right]$

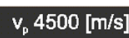

$\rho 3600\left[\mathrm{~kg} / \mathrm{m}^{3}\right]$ (b) \#1 - \#5

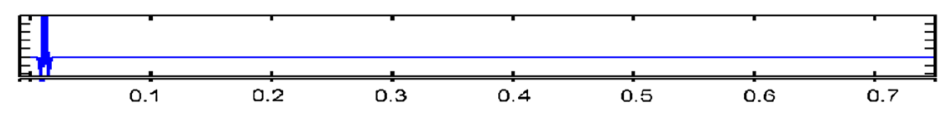

(c)

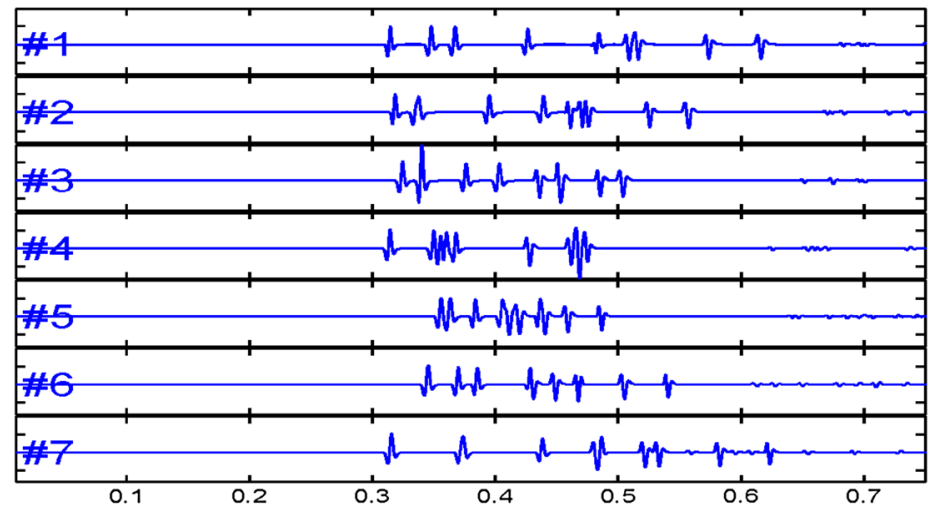

from sources (\#1-\#5) located in the bottom layer of the computational model (a) to the assumed receivers network (triangles \#1-\#7) located in the upper layer
Fig. 1 Computational model (a), assumed source function (b), and synthetic seismograms (c). Seismograms (c) were computed as a result of forward simulation of the seismic signals (b) propagated 
maximum stress components (Saenger 2011). With regard to the acoustic wave field modelling, the maximum absolute pressure value (MAPV) applied as an imaging condition appears to be a good indicator of the point source location (Eq. 2).

$\operatorname{MAPV}(x)=\max _{t \in T}|P(x, t)|$.

However, TRI using maximum pressure value can be problematic in the reconstruction in the seismic events that are not separated in time or space (Anderson et al. 2011).

The application of the PAPR coefficient is proposed in this paper to improve the spatial focus focal of seismic event detection.

The value of the PAPR coefficient can be computed from Eq. 3:

$\operatorname{PAPR}(x)=\frac{P_{\text {max }}^{2}(x)}{P_{\text {total }}^{2}(x) / T}, \quad P_{\text {total }}^{2}(x)=\sum_{t=1}^{T} P^{2}(x, t)$,

where $P_{\max }^{2}$ is the maximum energy of the signal recorded in the given computational node, $P_{\text {total }}^{2}$ is the total energy in the given node, $P(x, t)$ is the pressure value in each computational node, $t$ is time index, and $T$ is the number of computational time steps of the backward propagation of the seismic wave algorithm.

For both imaging conditions, the enormous values computed in the given computational node corresponding to the source point location are maintained during the whole process of backward wave propagations. Although both imaging parameters are calculated in much the same manner, the TRI with PAPR coefficient shows a higher spatial resolution that can improve the location of seismic event sequences.

\section{Numerical case study}

\section{Synthetic multiple source point simulations with the same emission time}

The results presented in this paper were obtained for an inhomogeneous, layered model. We assume that the $\mathrm{P}$ wave velocity increases from $3500 \mathrm{~m} / \mathrm{s}$ in the first layer, through $4200 \mathrm{~m} / \mathrm{s}$ in the second, up to $4500 \mathrm{~m} / \mathrm{s}$ in the third layer (Fig. 1a). For the numerical tests, the five sources with coordinates summarised in Table 1 and the same excitation time $t=0$ were used. The location of the sources is also depicted in Fig. 1a. The Ricker signal was used as a source function in the forward modelling (see Fig. 1b). The coordinates of the receivers are summarised in Table 2.

The receiver network reflects the locations and distances of surface seismic networks that are typical in Polish
Table 1 Coordinates of the source points assumed in numerical modelling

Table 2 Coordinates of receivers used in numerical simulations

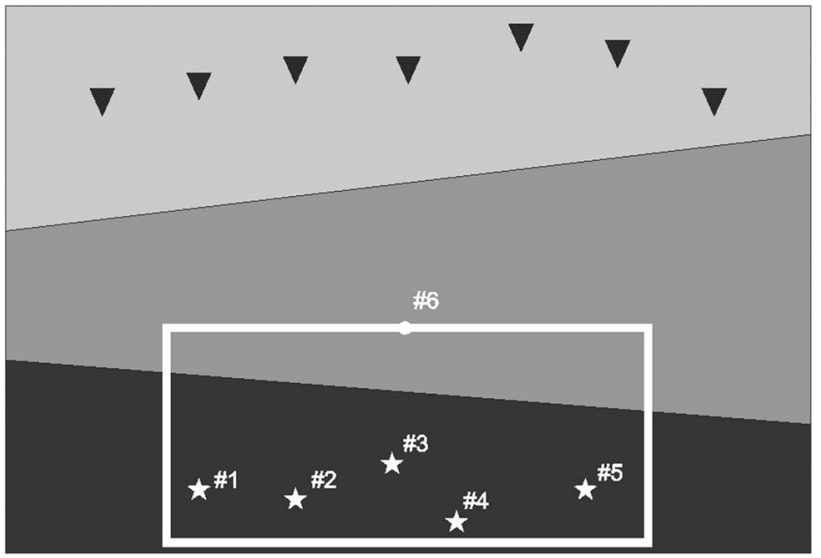

Fig. 2 Location of the analysis region (white rectangle) and the whole computational model

copper mines. Synthetic seismograms from these receivers computed in the forward simulation stage are presented in Fig. 1c.

The length of the source function is much shorter than the thickness of the geological layers in the assumed medium. As a result, the evaluated seismograms are mainly composed of isolated Ricker signals and interference between particular components is usually low (or medium for seismogram \#4). The highest amplitude components are direct waves and the waves reflected from the surface. The secondary waves are almost invisible in the seismograms.

Pressure changes and the PAPR ratio were computed for every node of the computational grid during the second stage of modelling when the time-reversed seismograms from Fig. 1c were used as source functions. 

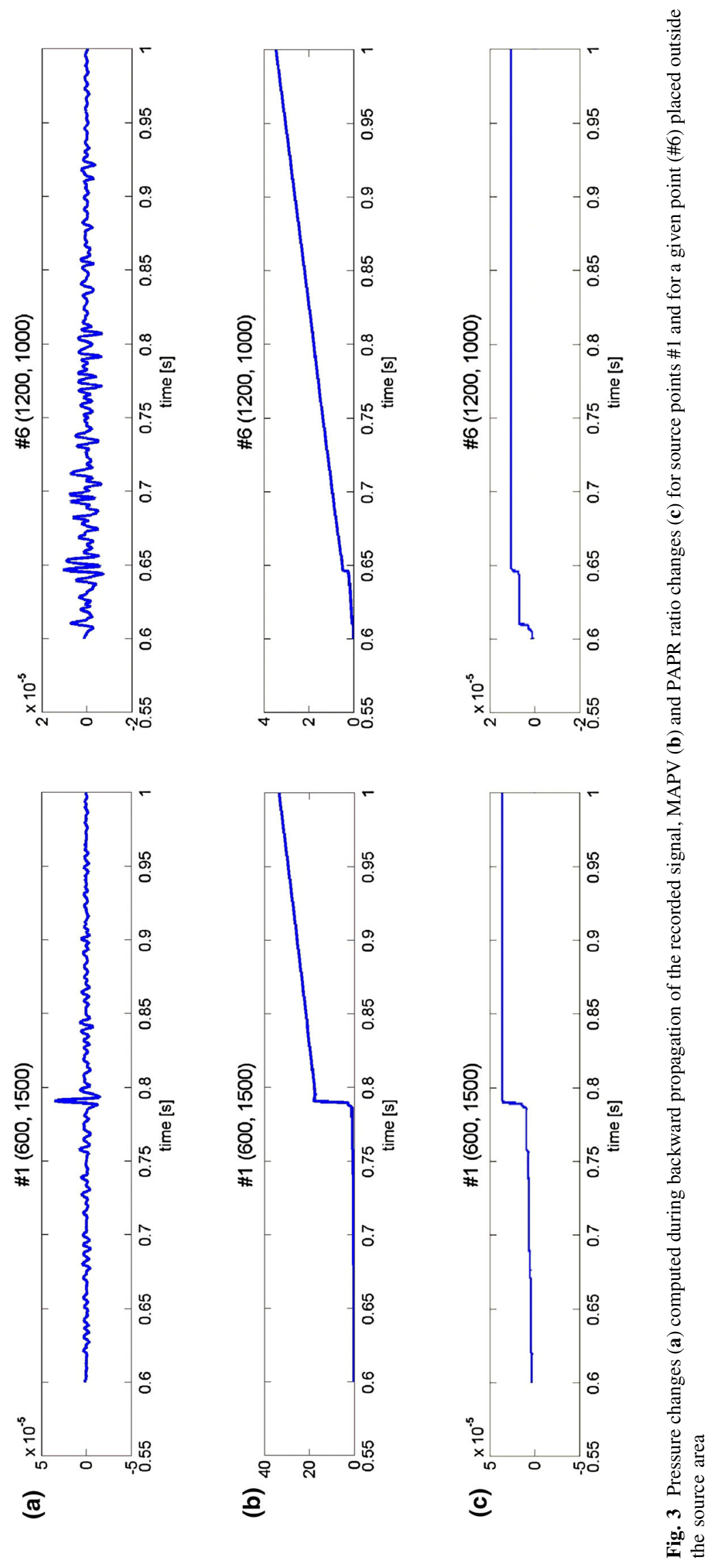

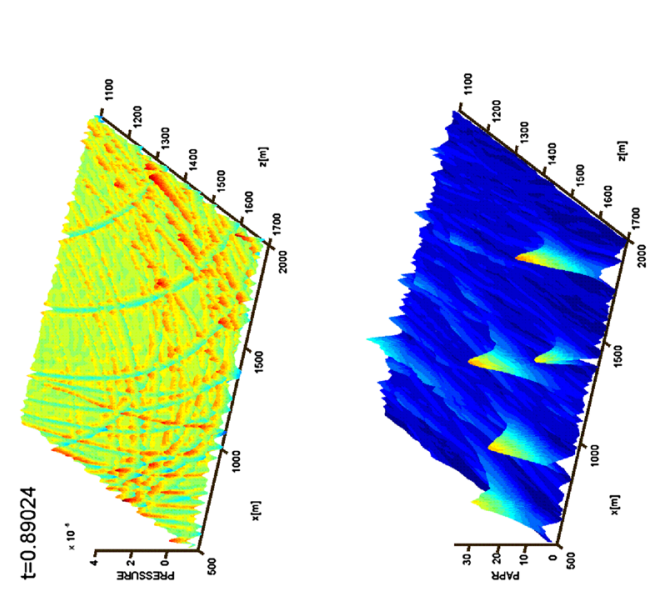

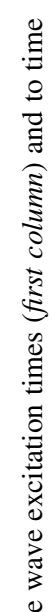
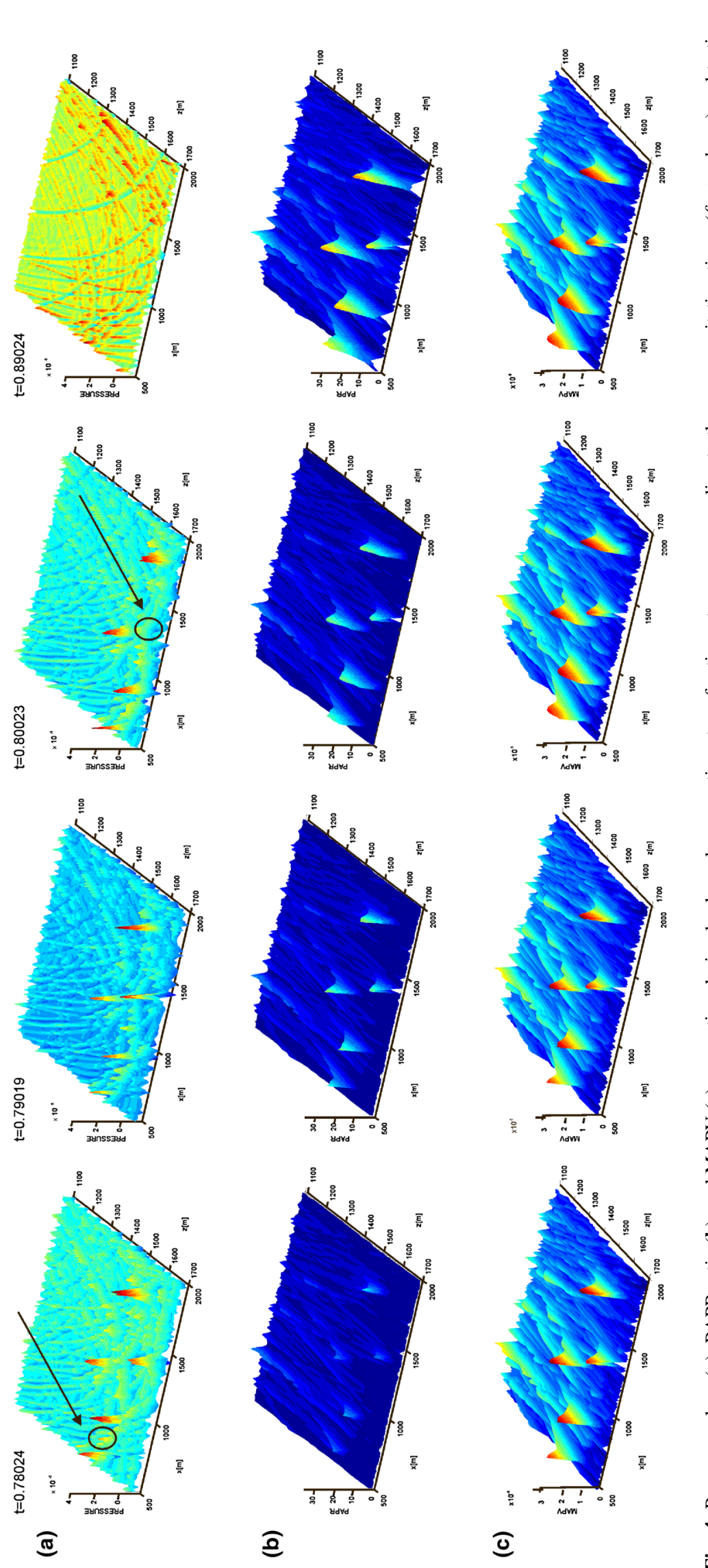

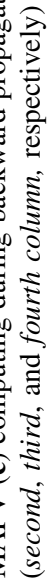

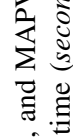

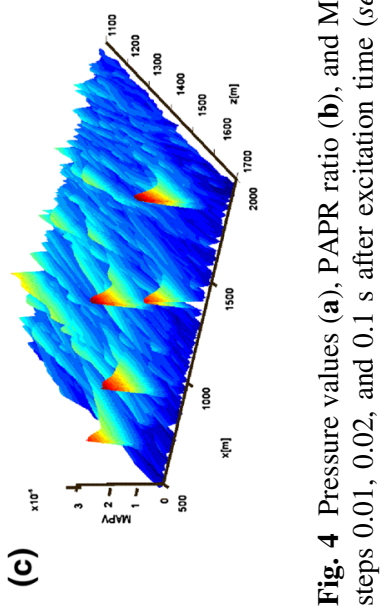




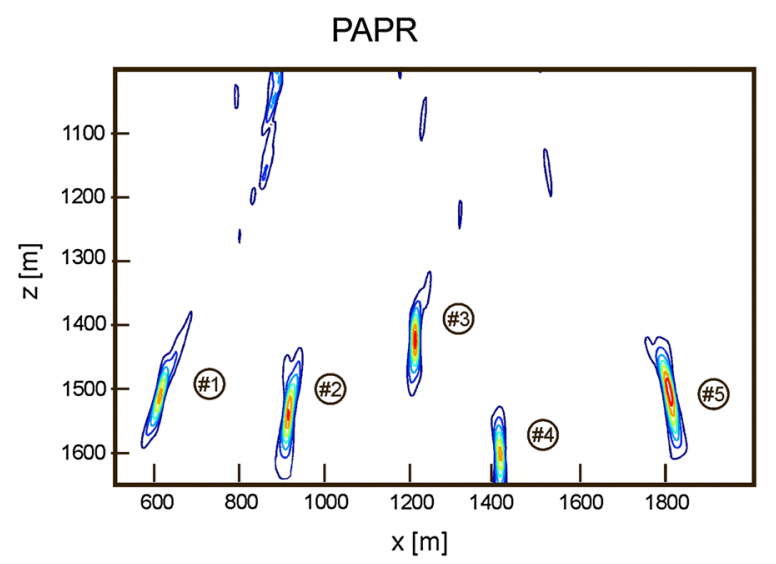

Fig. 5 The contour plot obtained after the last time step of the backward propagation stage, with areas of the highest values of PAPR ratio and MAPV normalised to the maximum values obtained during

\section{Quantitative assessment of focusing for multiple source point simulations with the same excitation time}

The analysis presented in the paper was limited to the area in the vicinity of seismic sources where we expected the focusing effect of the seismic waves. The location of the study area superimposed on the entire computational model is presented in Fig. 2.

The pressure, the PAPR ratio and MAPV changes in time obtained during backward propagation of the seismic wave are presented in Fig. $3 \mathrm{a}-\mathrm{c}$, respectively. Plots were prepared for the computational nodes corresponding to the assumed source point location \#1 (left column) and for one computational node placed in a source free point (plot \#6). The location of the computational nodes used in visualisation is also shown in Fig. 2.

For all types of plots, it is easy to indicate the moment of concentration of the energy at the source point (plot $\# 1$, left column in Fig. 3). For the seismic signal plotted for computational node placed outside the source point there are no such significant change (plot \#6, right column in Fig. 3). However, there are essential differences between pressure plots and plots of MAPV and PAPR value as MAPV and values of the PAPR ratio remains extremely large in the source point, whereas the increased pressure value fades over time. These relationships remain true for all the plots prepared for source points and for the plots prepared for the points that are outside the source area (Fig. 4).

The main difference between TRI using the PAPR ratio and TRI using MAPV is spatial resolution. As shown in Fig. 3b, the values achieved by the PAPR ratio in the source area are an order of magnitude greater than the values that it achieves outside this area. Values of MAPV outside the source area (Fig. 3c) are reduced only
MAPV

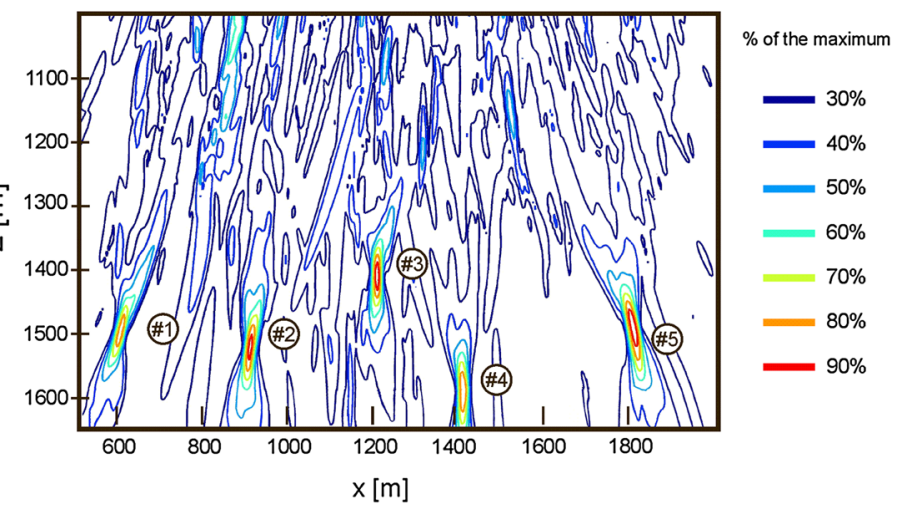

backward propagation of the seismic wave recorded for simulation of multiple sources emission

a few times in relation to the values that they take for the source area. In Fig. 5, the contour plots of the PAPR parameter values normalised to unity for given time steps are presented. Due to the transparency of the results, only values corresponding to $30,40, \ldots, 90 \%$ of the maximum value of the PAPR parameter are presented.

In both maps, only sources \#2, \#3, and \#4 are indicated by the contours corresponding to the $90 \%$ of the maximum value. Both TRI parameters correctly locate all the sources with the threshold of 80 and $70 \%$ of maximum value. In the normalised PAPR map, a few false locations appear only for the threshold of 40 and $30 \%$ of maximum value, whereas for this threshold noise hampering can be observed in the correct location in the normalised MAPV maps.

\section{Quantitative assessment of focusing for multiple source point simulations with different excitation times}

The advantage of TRI using the PAPR ratio value in the detection of coordinates of seismic sources was tested also for the model with different excitation times of seismic sources. Figure 6 presents plots for the computational model with interfering seismic signals. We assume the same numerical model of subsurface rock structure with the same P wave velocities as the previous case (Fig. 1a). The five sources with coordinates presented in Table 3 with different excitation times were used in the numerical tests. The location of the sources is also depicted in Fig. 6a. The excitation time of the seismic waves in the particular point sources were so chosen to imitate the rupture process (Udias et al. 2014). Synthetic seismograms computed during the forward simulation are presented in Fig. 6c. The Ricker signal assumed as a source functions in all five 
(a)

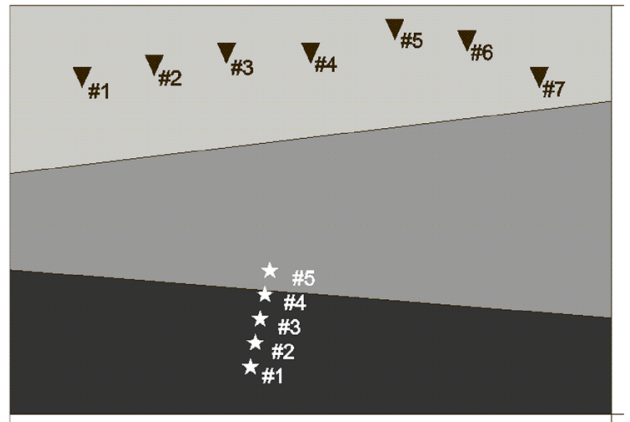

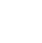

1700

Legend:

$\boldsymbol{\nabla}$ receiver

source

$\mathrm{v}_{\mathrm{p}} 3500[\mathrm{~m} / \mathrm{s}]$ $\rho 3500\left[\mathrm{~kg} / \mathrm{m}^{3}\right]$

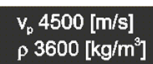

(b)

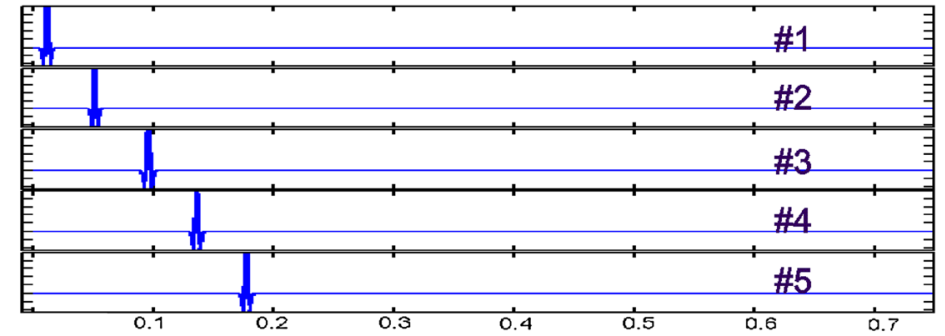

(c)

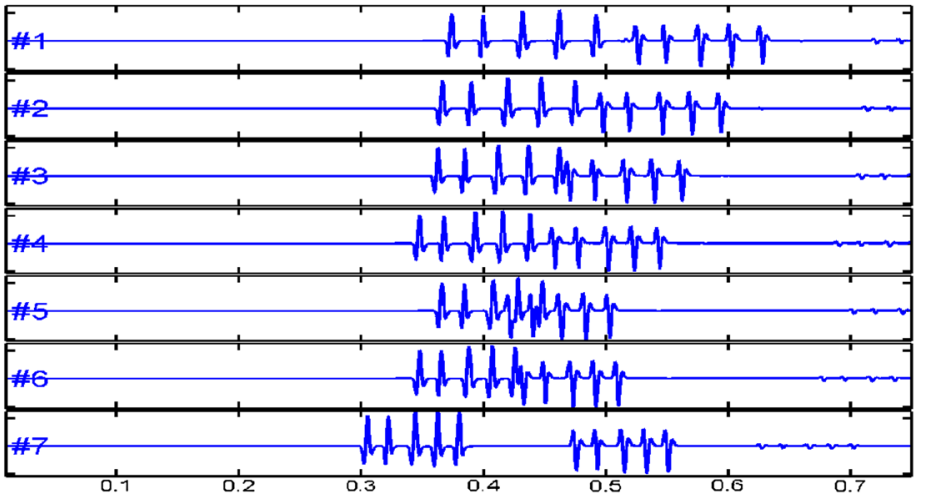

from sources (\#1-\#5) (a) to the assumed receiver network (triangles $\# 1-\# 7)$ located in the upper layer

sources. The differences appear for the contour of $60 \%$ of maximum value. In the MAPV maps, all five source areas are merged into one big area, whereas for the PAPR map such a low resolution is obtained only for the threshold of $30 \%$ of maximum value.

\section{Discussion and conclusions}

This paper introduces a new imaging condition that improved spatial resolution in the problem of seismic sources location. The application of PAPR ratio as an imaging condition improved the refocus for multiple sources with both the same excitation time and with a slight time lag between subsequent source emissions that simulate the rupture process. The results presented in this paper refer to synthetic data with a well-known subsurface structure velocity model. The almost perfect focusing obtained in the two examples presented above was achieved using detailed knowledge of the subsurface velocity structure and application of narrow broadband source signals (see Figs. 1b, 6b). For real data, only the first factor can be improved by adjustment of the numerical model of the subsurface structure to the real velocity model. The lack of the detailed velocity model is 
PAPR

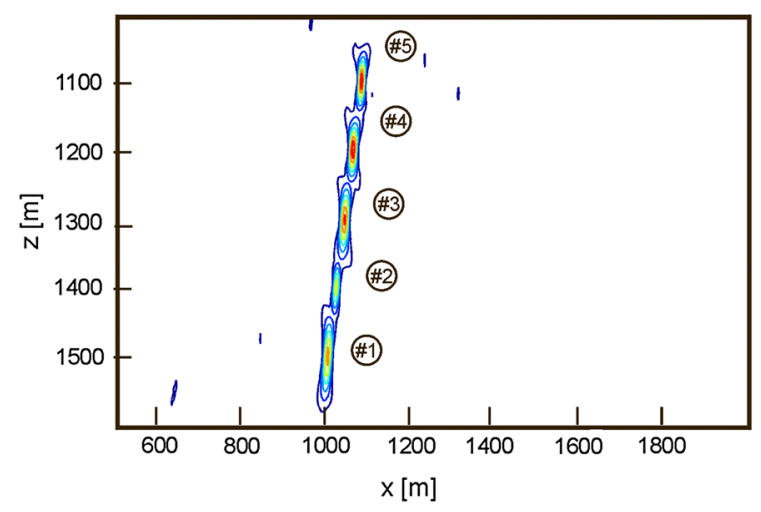

Fig. 7 The contour plot obtained after the last time step of the backward propagation stage, with areas of the highest values of PAPR ratio and MAPV normalised to the maximum values obtained during

the undeniable limitation of the application of the proposed imaging condition to the real recordings. This is the common factor negatively affecting all location procedures.

The results presented in this paper do not consider the presence of noise in the seismic records. Although the low values of the signal-to-noise ratio are regarded as one of the important conditions for correct seismic event location, the TRI using the PAPR ratio may be resistant to the presence of noise. The value of the PAPR ratio is the averaged value obtained in the given point, additionally decreasing in time. The noise is usually uncorrelated so the averaging procedure decreases its amplitude. Based on the above statements one can say that the use of the PAPR ratio coefficient can improve the location results comparing to other imaging coefficients used in the TRI.

It is important to mention that the source location as a function of space and time cannot be determined simultaneously. As shown in the article, the application of the PAPR ratio improves spatial location of seismic sources. Having better location of seismic sources, we can go back through the time snapshots to examine the time at which the amplitude achieves its maximum value: this is the origin time of the seismic event. It makes it possible to determine the time history of the source emissions, and time sequence of multiple sources.

The proposed method of TRI with PAPR ratio can be extended to elastic data. In this case, more imaging parameters can be used instead of the pressure value. The effects of geometry of the receiver network on the refocusing results of the multiple source emission are currently under investigation. The application of the proposed imaging condition to real data is also planned for the future.

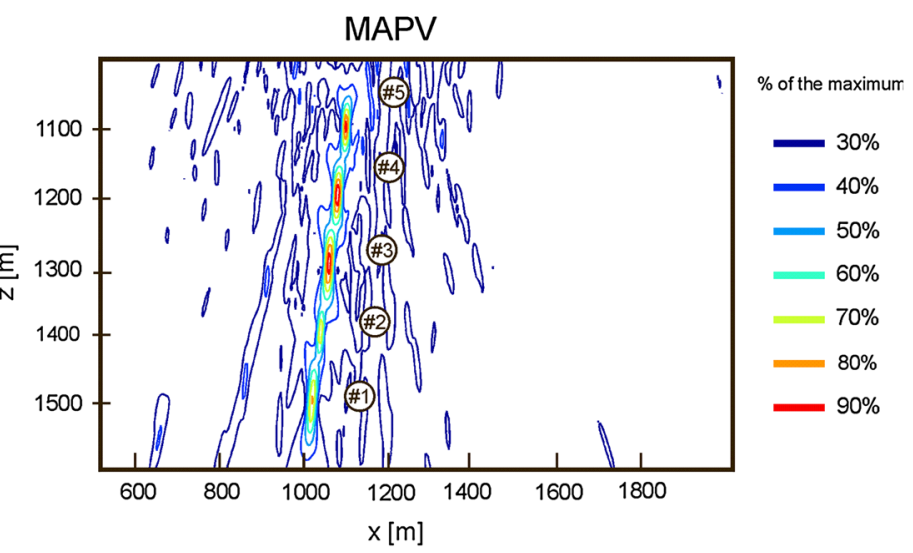

backward propagation of the seismic wave recorded for simulation of the rupture process

Acknowledgements This work was partly support by the AGH University of Science and Technology, Faculty of Geology, Geophysics and Environmental Protection, as a part of statutory Project 11.11.140.613. This paper was partially supported by Grant No. 2011/01/B/ST10/07305 from the National Science Centre, Poland.

Open Access This article is distributed under the terms of the Creative Commons Attribution 4.0 International License (http://crea tivecommons.org/licenses/by/4.0/), which permits unrestricted use, distribution, and reproduction in any medium, provided you give appropriate credit to the original author(s) and the source, provide a link to the Creative Commons license, and indicate if changes were made.

\section{References}

Anderson BE, Guyer RA, Ulrich TJ, Johnson PA (2009a) Time reversal of continuous-wave, steady-state signals in elastic media. Appl Phys Lett 94(11):111908. doi:10.1063/1.3097811

Anderson BE, Ulrich TJ, Griffa M, Le Bas P-Y, Scalerandi M, Gliozzi AS, Johnson PA (2009b) Experimentally identifying masked sources applying time reversal with the selective source reduction method. J Appl Phys 105(8):083506. doi:10.1063/1. 3079517

Anderson BE, Griffa M, Ulrich TJ, Johnson PA (2011), Time reversal reconstruction of finite sized sources in elastic media. J Acoust Soc Am 130(4):EL219-EL225. doi:10.1121/1.3635378

Artman B, Podladtchikov I, Witten B (2010) Source location using time-reverse imaging. Geophys Prospect 58(5):861-873. doi:10. $1111 / \mathrm{j} .1365-2478.2010 .00911$

Baysal E, Kosloff D, Sherwood JWC (1983) Reverse time migration. Geophysics 48(11):1514-1524. doi:10.1190/1.1441434

Blomgren P, Papanicolaou G, Zhao H (2002) Super-resolution in time-reversal acoustics. J Acoust Soc Am 111(1):230-248. doi: $10.1121 / 1.1421342$

Cerjan C, Kosloff D, Kosloff R, Reshef M (1985) A non-reflecting boundary condition for discrete acoustic and elastic wave equations. Geophysics 50(4):705-708. doi:10.1190/1.1441945

Debski W (2015) Using meta-information of a posteriori Bayesian solutions of the hypocentre location task for improving accuracy of location error estimation. Geophys J Int 201(3):1399-1408. doi:10.1093/gji/ggv083 
Debski W, Klejment P (2016) The new algorithm for fast probabilistic hypocenter locations. Acta Geophys 64(6):2382-2409. doi:10. 1515/acgeo-2016-0111

Douglas A (1967) Joint epicentre determination. Nature 215(5096):47-48. doi:10.1038/215047a0

Fichtner A, Bunge H-P, Igel H (2006) The adjoint method in seismology I. Theory. Phys Earth Planet Inter 157(1-2):86-104. doi:10.1016/j.pepi.2006.03.016

Fink M (1992) Time reversal of ultrasonic field-part I: basic principles. IEEE Trans Ultrason Ferroelectr Freq Control 39(5):555-566. doi:10.1109/58.156174

Fink M (1997) Time reversed acoustics. Phys Today 50(3):34-40. doi:10.1063/1.881692

Fink M, Prada C, Wu F, Cassereau D (1989) Self-focusing in inhomogeneous media with time reversal acoustic mirrors. IEEE Ultras Symp Proc 1(2):681-686. doi:10.1109/ULTSYM.1989. 67072

Gajewski D, Tessmer E (2005) Reverse modelling for seismic event characterization. Geophys J Int 163(1):276-284. doi:10.1111/j. 1365-246X.2005.02732.x

Hu LZ, McMechan GA (1988) Elastic finite difference modelling and imaging for earthquake sources. Geophys J Int 95(2):303-313. doi:10.1111/j.1365-246X.1988.tb00469.x

Kawakatsu H, Montagner J-P (2008) Time-reversal seismic source imaging and moment-tensor inversion. Geophys $\mathrm{J}$ Int 175(2):686-688. doi:10.1111/j.1365-246X.2008.03926.x

Kremers S, Fichtner A, Brietzke GB, Igel H, Larmat C, Huang L, Kaser M (2011) Exploring the potentials and limitations of the time reversal imaging of finite seismic sources. Solid Earth 2(1):95-105. doi:10.5194/se-2-95-2011

Larmat C, Montagner J-P, Fink M, Capdeville Y, Tourin A, Clévédé E (2006) Time-reversal imaging of seismic sources and application to the great Sumatra earthquake. Geophys Res Lett 33(19):L19312. doi:10.1029/2006GL026336

Larmat C, Guyer RA, Johnson PA (2010) Time-reversal methods in geophysics. Phys Today 63(8):31-35. doi:10.1063/1.3480073

Levander A (1988) Fourth-order finite-difference P-SV seismograms. Geophysics 53(11):1425-1436. doi:10.1190/1.1442422

McMechan G (1983) Migration by extrapolation of time-dependent boundary values. Geophys Prospect 31(3):413-420. doi:10.1111/ j.1365-2478.1983.tb01060.x

Parvulescu A, Clay CS (1965) Reproducibility of signal transmission in the ocean. Radio Electron Eng 29(4):223-228. doi:10.1049/ ree. 1965.0047
Rudzinski L, Debski W (2011) Extending the double-difference location technique to mining applications-part I: numerical study. Acta Geophys 59(4):785-814. doi:10.2478/s11600-0110021-5

Rudzinski L, Debski W (2012) Extending the double difference location technique-improving hypocenter depth determination. J Seismol. 17(1):83-94. doi:10.1007/s10950-012-9322-7

Saenger EH (2011) Time reverse characterization of sources in heterogeneous media. NDT E Int 44(8):751-759. doi:10.1016/j. ndteint.2011.07.011

Saenger EH, Kocur GK, Jud R, Torrilhon M (2011) Application of time reverse modelling on ultrasonic non-destructive testing of concrete. Appl Math Model 35(2):807-816. doi:10.1016/j.apm. 2010.07.035

Steiner B, Saenger EH (2010) Comparison of 2D and 3D time reverse modelling for tremor source localization. SEG Tech Program Expand Abstr 2010:2171-2175. doi:10.1190/1.3513275

Steiner B, Saenger EH (2012) Comparison of 2D and 3D time-reverse imaging - a numerical case study. Comp Geosci 46:174-182. doi:10.1016/j.cageo.2011.12.005

Steiner B, Saenger EH, Schmalholz SM (2008) Time reverse modelling of low-frequency microtremors: application to hydrocarbon reservoir localization. Geophys Res Lett 35(3):L03307. doi:10.1029/2007GL032097

Tarantola A (1988) Theoretical background for the inversion of seismic waveforms, including elasticity and attenuation. Pure Appl Geophys 128(1):365-399. doi:10.1007/BF01772605

Udias A, Madariaga R, Buforn E (2014) Source mechanisms of earthquakes. Theory and practice. Cambridge University Press, Cambridge

Virieux J (1986) P-SV wave propagation in heterogeneous media: velocity-stress finite-difference method. Geophysics 51(4):889-901. doi:10.1190/1.1442147

Waldhauser F, Ellsworth W (2000) A double-difference earthquake location algorithm: method and application. Bull Seismol Soc Am 90(6):1353-1368. doi:10.1785/0120000006

Willis ME, Lu R, Burns DR, Toksoz MN, Campman X, de Hoop M (2006) A novel application of time reversed acoustics: salt dome flank imaging using walk away VSP surveys. Geophysics 71(2):A7-A11. doi:10.1190/1.2187711 